\title{
Fatigue Analysis of FRP Strengthened RC Slabs Reinforced with Plain Bars Under Moving Load
}

\author{
Ahmed Attia M. Drar ${ }^{1(\bowtie)}$ and Takashi Matsumoto ${ }^{2}$ \\ ${ }^{1}$ Sohag University, Sohag, Egypt \\ attya85@yahoo.com \\ 2 Hokkaido University, Sapporo, Japan \\ takashim@eng.hokudai.ac.jp
}

\begin{abstract}
In Japan, old RC bridge-deck slabs were economically designed by small thickness without considering the fatigue resistance. These slabs are subjected to a huge repetition of moving loads. Therefore, they are suffering from fatigue damage. This damage is more significantly observed than that slabs reinforced with deformed bars. To extend their fatigue life, a suitable strengthening technique is required such as externally bonded FRP sheets. Using numerical method for predicting the improvement in their fatigue life is strongly beneficial to take full advantage of this strengthening technique. This study presents a proposed numerical method based on bridging stress degradation concept to analyze two full scale RC slabs reinforced with plain bars under moving load. One of them is strengthened with externally bonded FRP sheets in longitudinal and transverse directions on the slab bottom surface. The interfacial bond behavior between FRP sheet and concrete surface with its degradation due to fatigue loading is implemented. This study provides the propagation of cracked elements, center displacement evolution, cracking pattern and FRP strain. For the strengthened RC slab, the major crack opening is restricted by the contribution of FRP sheets in longitudinal and transverse directions. Therefore, the strengthened RC slab shows longer fatigue life and smaller deformation. By comparing these numerical results with the experimental results, the current numerical method provides a good agreement.
\end{abstract}

\section{Introduction}

Reinforced concrete (RC) bridge is one of the most common structural members, which is subjected to a large number of load repetitions due to heavy traffic resulting a fatigue damage. This damage is significantly observed in the RC slab than other member. Therefore, it is important to consider the fatigue resistance in the design. According to previous studies of Matsui (1987); and Perdikaris and Beim (Perdikaris and Beim 1988), the most common mode of fatigue failure was by punching shear mode, which is significant in RC slab with small thickness.

Old slabs in Japan are suffering from two main problems as the follows. First problem is that these slabs were economically designed with small thickness. Second problem is that the plain bars were used as reinforcement for some of these slabs. Therefore, 
a significant fatigue damage was observed in theses slabs than those reinforced with deformed bars (Shakushiro et al. 2011). Therefore, a suitable strengthening technique is required to extend their fatigue life such as externally bonded fiber reinforced polymer (FRP), which have been extensively used for external strengthening of RC structure members. Mitamura et al. (2011) provided an experimental study to evaluate the effect of FRP strengthening on the fatigue durability improvement of RC slabs reinforced with plain bars under moving load fatigue. But, non-perfect-strengthening design causes a loss in the functions of the structures over time. Therefore, the main target of this study is developing numerical method to evaluate this strengthening and predict the extended fatigue life for RC slabs reinforced with plain bars.

For RC slabs reinforced with plain bars, the proposed numerical method by Drar et al. (2015); and Drar and Matsumoto (2016) was employed to predict their fatigue life and behaviors. Therefore, this study can be extended to examine the effect of FRP strengthening on these slabs. The experimental study by Mitamura et al. (2011) can be used to verify the numerical results.

\section{Method}

In this section, the developed numerical method will be described. This method was developed based on the finite element method (FEM) considering the different materials behaviors under the fatigue loading. Therefore, the following explanation shows the numerical model for all components of the FRP composite structure.

\subsection{Concrete}

Concrete is different than other homogenous materials because it contains a mixture of different components with different stiffness. Therefore, it exhibits a large number of micro cracks at the interface between the aggregates and mortar resulting a nonlinear behaviour. Eight-node three-dimensional (3D) smeared crack elements with multiple fixed crack concept by Rots and Blaauwendraad (1989) is employed to model concrete elements. Table 1 shows the constitutive laws of concrete (Maekawa et al. 2003).

Table 1. Constitutive laws for concrete (Maekawa et al. 2003)

\begin{tabular}{l|l|l|l}
\hline \multicolumn{2}{l|}{ Compression } & \multicolumn{1}{l}{ Tension } \\
\hline$\varepsilon_{m} \leq \varepsilon \leq 0$ & $\sigma=f_{c} \frac{\varepsilon}{\varepsilon_{m}}\left(2-\frac{\varepsilon}{\varepsilon_{m}}\right)$ & $0 \leq \varepsilon \leq \varepsilon_{t}$ & $\sigma=E_{c} \varepsilon$ \\
\hline$\varepsilon_{u} \leq \varepsilon<\varepsilon_{m}$ & $\sigma=f_{c} \frac{\varepsilon_{u}-\varepsilon}{\varepsilon_{u}-\varepsilon_{m}}$ & $\varepsilon_{t}<\varepsilon$ & $\sigma=f_{t}\left(\frac{\varepsilon_{t}}{\varepsilon}\right)$ \\
\hline$E_{c}=$ the modulus of elasticity of concrete, $f_{c}=$ concrete \\
compressive strength, $\varepsilon_{m}=f_{c} / 2 E_{c}=$ concrete strain \\
corresponding to $f_{c}, f_{t}=$ tensile strength, \\
$\varepsilon_{t}=f_{t} / E_{c}=$ strain at tensile strength, and $\varepsilon_{u}=2$ \\
$\varepsilon_{m}=$ crushing strain.
\end{tabular}




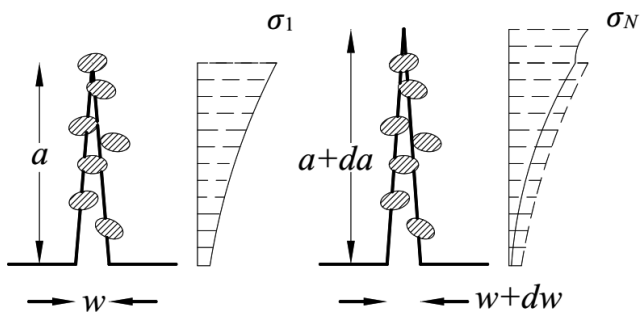

Fig. 1. Crack propagation due to bridging stress degradation at first cycle and after $N$ cycles (Drar and Matsumoto 2016)

To simulate the cracking behavior of concrete, the bridging stress is employed. The bridging stress is the transferred stress between crack surfaces through aggregates. When fatigue loading is applied, the crack is subjected to a process of opening and closing. This leads to reduce the bridging stress in cracked concrete. This reduction can be defined as bridging stress degradation concept. This degradation leads to a localized increase of stress at crack tip resulting an increase of crack length and its width as shown in Fig. 1. The bridging stress degradation for cracked concrete can be expressed by the following equation (Jun and Stang 1998; Li and Matsumoto 1998).

$$
\frac{\sigma_{N}}{\sigma_{1}}=1-\left(0.08+4 \cdot \varepsilon_{t \max } l\right) \log (N)
$$

where $l$ is cracked element size, $\varepsilon_{t \max }$ maximum tensile strain, $N$ is number of cycles, $\sigma_{N}$ and $\sigma_{1}$ are bridging stress at the $N$ th and the first cycle, respectively.

\subsection{Reinforcing Bar}

To model the reinforcement of RC slab elements, the smeared rebar concept is used according to its reinforcement ratio in any direction. Bilinear stress-strain relationship is employed for reinforcing bar model with explicit yield stress, $f_{y}$. For all slabs, the plain reinforcing bars were used in the upper and lower reinforcement. Therefore, it is important to accurately model the bond-slip effect between the plain reinforcing bar and its surrounding concrete. This effect is considered by adding equivalent bond strain to plain bar strain. This leads to decrease the reinforcing bar stiffness as shown in Fig. 2. The modified reinforcing bar stiffness can be defined as effective stiffness, $E_{s}^{*}$, which can be calculated from the following equations

$$
\begin{gathered}
E_{s}^{*}=\frac{f_{y}^{*}}{\varepsilon_{y}^{*}}, \\
\varepsilon_{y}^{*}=\varepsilon_{y}+\frac{S_{y}}{l_{d}},
\end{gathered}
$$




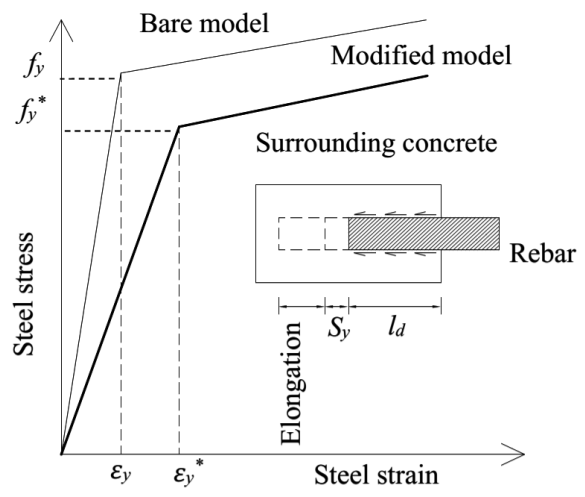

Fig. 2. Modified plain reinforcing bar model (Drar and Matsumoto 2016)

where $f_{y}^{*}$ is the effective yield stress (Belarbi and Hsu 1994), $S_{y}$ is the slip displacement of a steel bar at yield point (Zhao and Sritharan 2007), $l_{d}$ is the transmission length of bond stress, $\varepsilon_{y}$ and $\varepsilon_{y}^{*}$ are the explicit and effective yield strain, respectively.

\section{FRP}

A linear elastic stress-strain relation is employed to model FRP elements. This assumption is good enough to simulate FRP behavior because reaching to its maximum tensile strength is assumed to be very difficult. The reason is that the maximum tensile strength of the FRP is much larger than other materials. Moreover, the authors assumed that there is no strength degradation due to fatigue loading (Loo et al. 2012).

\subsection{Interfacial Bond Element}

Adhesive material is used to bond FRP sheets with the facing concrete surface, which is considered as the weakest part of FRP composite. Therefore, the interfacial bond behavior between FRP sheet and concrete surface and its degradation due to fatigue loading are carefully integrated to obtain accurate numerical results. Their numerical model can be explained as follows.

The mechanical behavior of the interfacial bond element can be simulated as a relationship between the local bond stress, $\tau$, and the relative slip displacement, $S$. The interfacial fracture energy, $G_{f}$, can be represented by the area under the $\tau-S$ curve, which corresponds to the energy per unit bond area required for complete debonding. A bilinear bond-slip relation ( $\mathrm{Lu}$ et al. 2005, 2004) is used in the present study as shown in Fig. 3. This relationship can be divided to three parts; (1) before debonding, (2) debonding initiation, and (3) complete debonding. The maximum bond strength, $\tau_{\max }$, the corresponding slip, $S_{0}$, and the total fracture energy, $G_{f}$, are governed by the tensile strength of the concrete, $f_{t}$, and a width ratio parameter, $B$, as follows; 


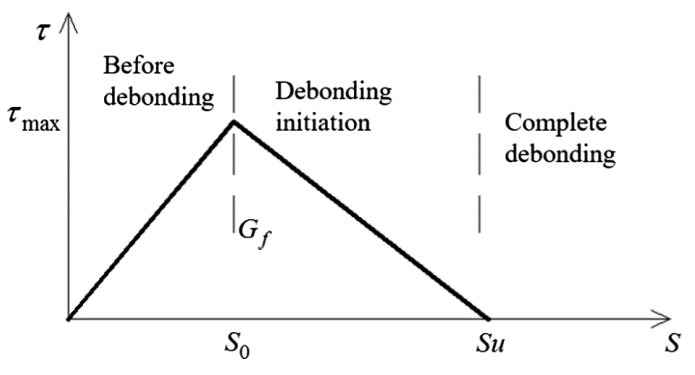

Fig. 3. Interfacial bond-slip model

$$
\begin{gathered}
\tau_{\max }=1.5 \cdot B \cdot f_{t}, \\
S_{0}=0.0195 \cdot B \cdot f_{t}, \\
G_{f}=0.308 \cdot B^{2} \cdot \sqrt{f_{t}}, \\
B=\sqrt{\frac{2.25-b_{f} / b_{c}}{1.25+b_{f} / b_{c}}},
\end{gathered}
$$

where $b_{f} / b_{c}$ is the ratio of total FRP laminate width, $b_{f}$, to the total slab width, $b_{c}$.

This bond-slip model will be degraded in the case of fatigue loading. The bond stress, $\tau$, versus slip displacement, $S$, relationship by Dai et al. (2005) shows a decrease of interfacial bond stiffness and an increase of the slip displacement. In this study, the model by Loo et al. (2010) is employed by reducing the interfacial bond stiffness according to the following equation.

$$
E_{b}=E_{b 0}\left[1+\alpha(\log N)^{\beta} \cdot\left(\frac{\Delta \tau}{\tau_{f}}\right)^{\gamma}\right],
$$

where $E_{b}$ is the interfacial bond stiffness at the number of cycles $N, E_{b 0}$ is the interfacial bond stiffness at the first cycle, $\Delta \tau$ is the bond stress range, $\tau_{f}$ is the bond stress at failure, and the constants $\alpha, \beta$ and $\gamma$ are parameters to fit the experimental data of Dai et al. and Yun et al. (Dai et al. 2005; Yun et al. 2008). $\alpha, \beta$ and $\gamma$ equal to $-190.3,0.990$ and 8,797, respectively.

For simplicity, uniform interfacial bond stiffness, $E_{b}$, is used for all bond elements according to the biggest bond stress range, $\Delta \tau$. This simplification can be considered as the worst case to simulate the fatigue life. Figure 4 shows the degradation of interfacial bond stiffness due to fatigue loading. 


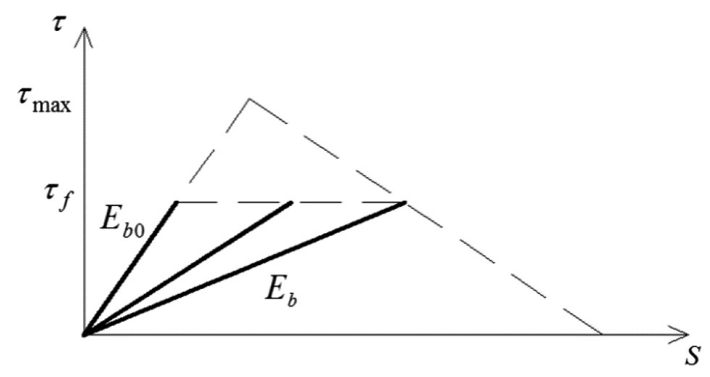

Fig. 4. The degradation of interfacial bond stiffness due to fatigue loading

\section{Fatigue Analysis}

This numerical method is used to analyze two full-scale slabs. One of them is strengthened with externally bonded FRP sheets in longitudinal and transverse directions on the slab bottom surface. These slabs are verified with the experimental results by Mitamura et al. (2011). The details of analyzed RC slabs with the dimensions of FRP sheets is shown in Table 2. MSC Marc-Mentat is used to analyze theses slabs with integrated Fortran subroutine, which considers the bridging stress degradation of concrete by increasing number of cycles. All slabs were supported by two steel I-beams over their longitudinal direction to simulate an elastic reaction behavior. In their transverse direction, these slabs were simply supported by hinged support. The moving load was applied in the longitudinal direction by two wheels with the width of $190 \mathrm{~mm}$ for each wheel. The moving load level is increased with increasing number of cycles according to Table 3. Figure 5 shows slab geometry and arrangement of reinforcing bars. The materials properties and loading pattern of these slabs are the same as those used in the experimental study. Plain reinforcing bars are used for reinforcing these slabs. Table 4 shows The material properties. Figure 6 shows the numerical model for strengthened RC slab, S330, with FRP sheets arrangement.

Table 2. Details of analyzed RC slabs

\begin{tabular}{l|l|l|l|l|l}
\hline Slab & Slab dimensions & \multicolumn{4}{|l}{ FRP sheets } \\
\cline { 3 - 6 } id & $(\mathrm{mm})$ & $\begin{array}{l}\text { Width } \\
(\mathrm{mm})\end{array}$ & $\begin{array}{l}\text { Thickness } \\
(\mathrm{mm})\end{array}$ & $\begin{array}{l}\text { Longitudinal } \\
\text { spacing }(\mathrm{mm})\end{array}$ & $\begin{array}{l}\text { Transverse } \\
\text { spacing }(\mathrm{mm})\end{array}$ \\
\hline S0 & $3300 \times 2650 \times 160$ & \multicolumn{4}{|l}{ Without strengthening } \\
\cline { 3 - 6 } & & 100 & 1.2 & 330 & 330 \\
\hline
\end{tabular}

Table 3. Moving load levels for analyzed RC slabs

\begin{tabular}{l|l|l|l|l|l|l}
\hline Number of cycles $\left(\times 10^{5}\right.$ cycles $)$ & $>1$ & $1-2$ & $2-3$ & $3-4$ & $4-7.8$ & $>7.8$ \\
\hline Moving load $(\mathrm{kN})$ & 120 & 130 & 150 & 170 & 200 & 230 \\
\hline
\end{tabular}




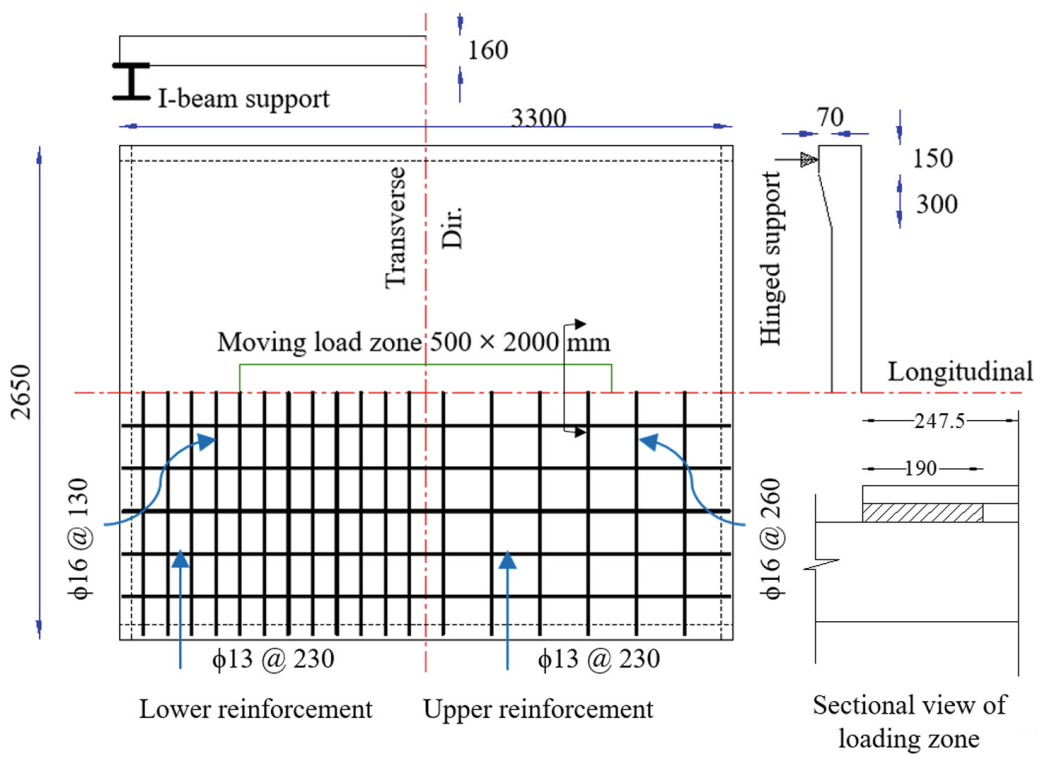

Fig. 5. RC slab geometry and the arrangement of reinforcing bars (all dimensions in $\mathrm{mm}$ )

Table 4. Materials properties (Mitamura et al. 2011)

\begin{tabular}{l|l|l|l|l}
\hline Material & Young's modulus (GPa) & Poisson's ratio & \multicolumn{2}{|l}{ Strength (MPa) } \\
\cline { 4 - 5 } & & & Tension & Compression \\
\hline Concrete & 26.133 & 0.2 & 3.3 & 45.26 \\
\hline Reinforcing bar & 187 & 0.3 & 235 (Yield) \\
\hline FRP & 171 & 0.3 & 3320 & - \\
\hline Adhesive & 2.66 & 0.3 & 35 & 56 \\
\hline
\end{tabular}

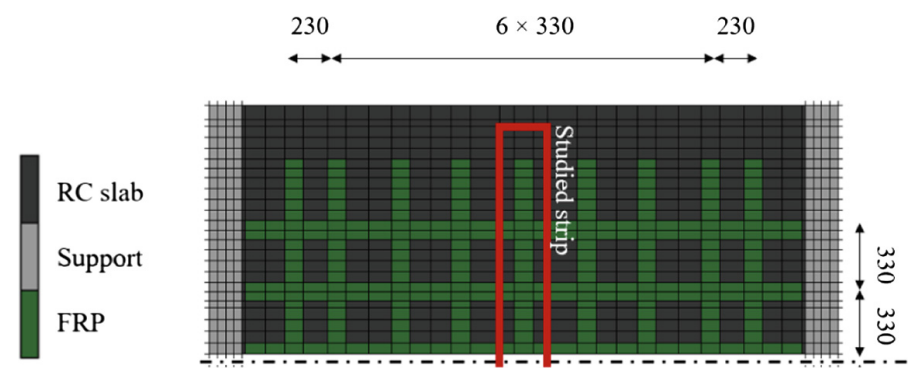

Fig. 6. FRP sheets arrangement for strengthened RC slab S330 


\subsection{Propagation of Cracked Elements}

The propagation of cracked elements of the RC slabs at different numbers of cycles is shown in Fig. 7. According to the geometrical symmetry in both directions, one fourth of a slab model is shown in this figure to investigate the propagation of internal cracked elements in longitudinal and transverse directions. White-colored indicates to non-cracked elements. The cracked zone caused by the first cycle is indicated by blue. The cracked zones caused by further cycles are indicated by other colored elements. The propagation of cracked elements in the transverse direction (T.D.) is extended from the center of the loading area. For the longitudinal direction (L.D.), the cracked elements are distributed over the movement zone due to the movement effect of the applied moving load along this direction.

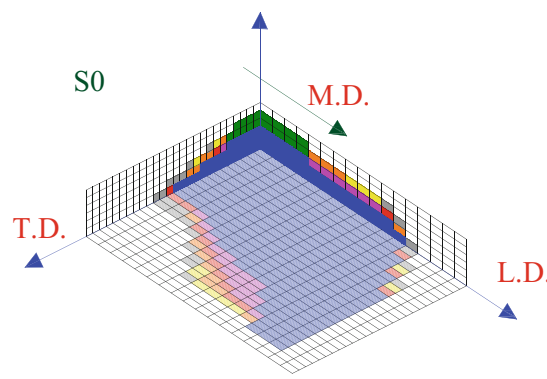

Cycles 辰 $1 \square 10 \square 10^{3} \square 10^{4} \square$

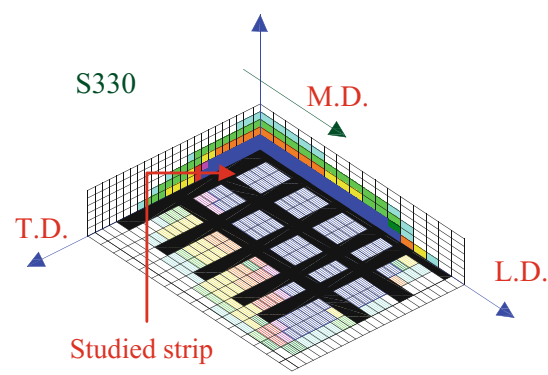

$2 \times 10^{5}$

$8 \times 10$

Fig. 7. Propagation of cracked elements for analyzed RC slabs

At first cycle, strengthened RC slab, S330, shows a smaller cracked zone with smaller thickness than the non-strengthened RC slab, S0. By increasing number of cycles, the cracked elements propagated due to the bridging stress degradation concept. Table 5 shows the percentages of cracked elements volume. Strengthened RC slab, S330, shows a smaller volume percentage than the non-strengthened RC slab, S0, at first cycles. These percentages are increased with increasing number of cycles. At fatigue failure, the percentages of cracked elements volume for all slabs are almost same. The average degradation ratio can be defined as the ratio of the difference between the cracked elements volume at fatigue failure and first cycle by $\mathrm{mm}^{3}$ to the fatigue life by cycles. The average degradation ratios for all analyzed slabs are listed in Table 5. Strengthened RC slab, S330, shows a slower degradation ratio than the non-strengthened RC slab, S0. The reason is that the FRP strengthening plays an important role to restrict the major crack opening. This leads to decrease the maximum tensile strain of concrete than the non-strengthened RC slab resulting in slower bridging stress degradation according to Eq. (1). 
Table 5. The percentages of cracked elements volumes

\begin{tabular}{l|l|l|l|c}
\hline \multirow{2}{*}{ Slab id } & \multicolumn{3}{|l|}{ Cracked elements volume \% } & \multirow{2}{*}{ Average degradation ratio $\left(\mathrm{mm}^{3} /\right.$ Cycle $)$} \\
\cline { 2 - 4 } & $N=1$ & $N=400,000$ & $N=890,000$ & \\
\hline S0 & $22 \%$ & $49.1 \%$ & & 9479.6 \\
\hline S330 & $10.7 \%$ & $33.6 \%$ & $50.5 \%$ & 618.8 \\
\hline
\end{tabular}

\subsection{Center Displacement Evolutions}

Center displacement evolution versus the number of cycles for all analyzed slabs compared with the experimental results is shown in Fig. 8. Increasing number of cycles results in an increase of center displacement. The reason is that the propagation of cracked elements in Fig. 7 leads to decrease RC slab stiffness due to the concept of bridging stress degradation. Moreover, increasing moving load level leads to a rapid increase of center displacement due to the increase of RC elements strain.

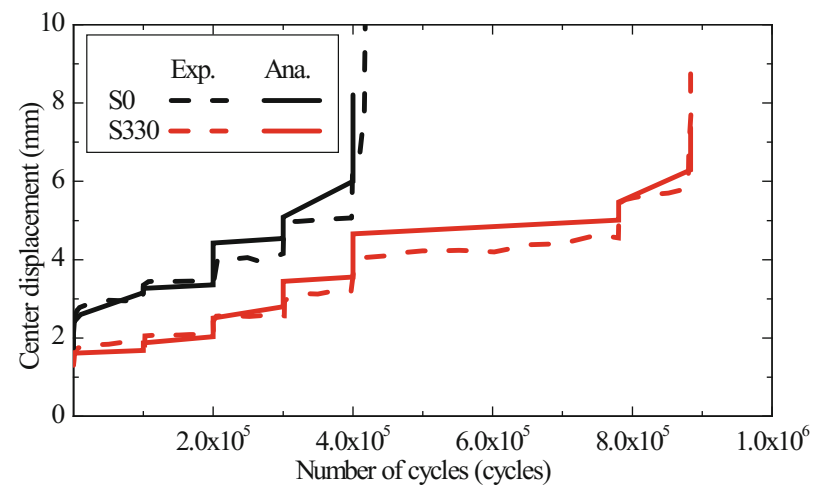

Fig. 8. Center displacement evolutions

Strengthened RC slab, S330, shows a smaller center displacement than the non-strengthened RC slab, S0, at the same number of cycles. The reason is that FRP strengthening works as an additional reinforcement for strengthened RC slab. This leads to a decrease of concrete strain. Moreover, center displacement evolution of non-strengthened RC slab, S0, shows a higher slope than that in strengthened RC slab, S330. Therefore, the reduction ratio of slab stiffness for non-strengthened RC slab, S0, is larger. The possible reason can be explained as that the FRP strengthening leads to a significant slower degradation ratio than the non-strengthened RC slab as shown in Table 5. According to this explanation, strengthened RC slab, S330, shows a longer fatigue life than the non-strengthened RC slab.

The comparison between numerical and experimental center displacement evolution provides similar values for fatigue life and center displacement, indicating an acceptable agreement between them. 


\subsection{Transverse FRP Sheet Strain}

The numerical and experimental transverse strain distributions of FRP sheet at slab center for strengthened RC slab, S330, at different moving load levels is shown in Fig. 9. The location of longitudinal FRP sheets is indicated by dashed-green box to observe their effect on the center transverse sheet. The location of studied FRP sheet in transverse direction is indicated by red box in Fig. 6. The horizontal axis shows the transverse length starting from slab center.

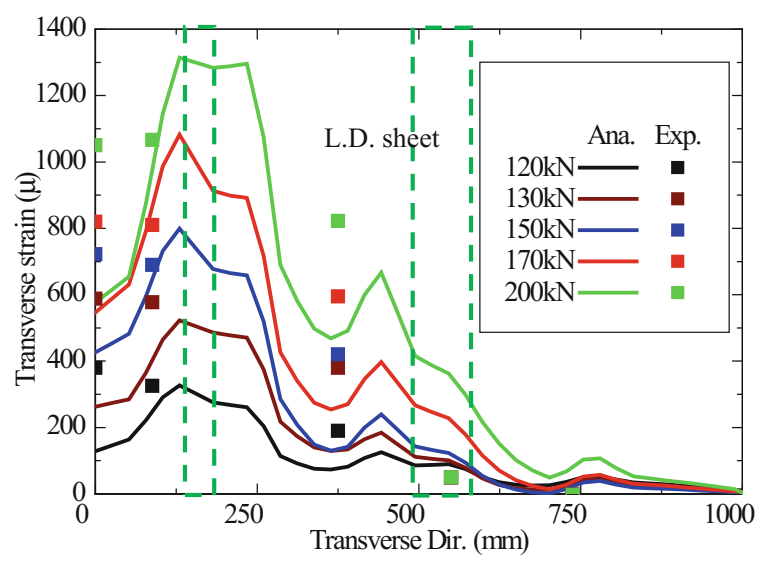

Fig. 9. Transverse FRP sheet strain at slab center for strengthened RC slab S330

The maximum numerical and experimental strain values located at the loading position. Moreover, increasing moving load level leads to increase numerical and experimental FRP sheet strain. This increasing is significant at the loading position than other parts. The reason is that the bridging stress degradation of concrete is influenced by the maximum tensile strain, which locates in this zone. This leads to crack opening localization at slab center resulting in a higher strain of surrounding FRP sheets. Therefore, the improvement of FRP strengthening is significant at slab center, which contains a wide crack opening for the major cracks.

Transverse FRP strain shows a significant decreasing at the overlapping location with the longitudinal sheets in the numerical results. The reason is that the tensile force in perpendicular sheet leads to a strain shrinkage at overlapping location as shown in Fig. 10. This leads to a difference between the numerical and experimental results at overlapping location. This observation cannot be shown in the experimental results due to the effect of separated layers with different strain for each layer. The overlapping element between longitudinal and transverse sheets shares the same strain distribution in the numerical analysis. In other parts, the good agreement between numerical and experimental results is provided. Moreover, the extension lines between numerical results pass the experimental results indicating an acceptable agreement between them in the overlapping locations. 


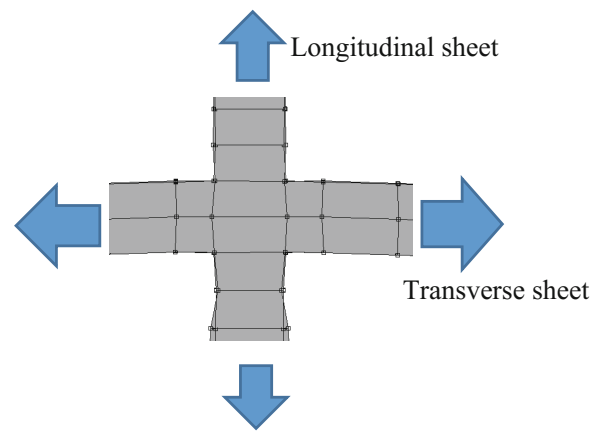

Fig. 10. Longitudinal and transverse FRP sheets overlapping

\section{Conclusions}

A proposed numerical method based on bridging stress degradation concept was presented in this paper to study the effect of FRP strengthening on the fatigue behaviors of $\mathrm{RC}$ slabs reinforced with plain bars. The interfacial bond behavior between FRP sheet and facing concrete surface with its degradation due to fatigue loading was considered in this method to obtain accurate numerical results. The comparison between numerical and experimental results provided an acceptable agreement between them.

Strengthened RC slab provided a longer fatigue life and smaller deformations than the non-strengthened RC slab. To investigate this improvement, the numerical analysis was conducted in this study. FRP strengthening leads to restrict the major crack opening, which is distributed due to moving load fatigue. This results in a slower degradation ratio than the non-strengthened RC slab.

FRP sheet strain showed a localized higher strain at slab center, which contain a wide crack opening due to significant bridging stress degradation in this zone. Therefore, an extensive FRP strengthening at slab center is recommended.

\section{References}

Belarbi, A., Hsu, T.T.: Constitutive laws of concrete in tension and reinforcing bars stiffened by concrete. Struct. J. 91, 465-474 (1994)

Dai, J.G., Saito, Y., Ueda, T., Sato Y.: Static and fatigue bond characteristics of interfaces between CFRP sheets and frost damage experienced concrete. In: Proceedings of Fourth International Symposium on Fiber Reinforced Polymer Reinforcement for Reinforced Concrete Structures (FRPRCS-4), pp. 1515-1530 (2005)

Drar, A.A.M., Matsumoto, T.: Fatigue analysis of RC slabs reinforced with plain bars based on the bridging stress degradation concept. J. Adv. Concr. Technol. 14, 21-34 (2016)

Drar, A.A.M., Matsumoto, T., Hayashikawa, T., He, X.: Development of a numerical model to predict the fatigue behaviors of RC slabs. J. Struct. Eng. JSCE 71, I_805-I_812 (2015)

Jun, Z., Stang, H.: Fatigue performance in flexure of fiber reinforced concrete. Mater. J. 95, 5867 (1998) 
Li, V.C., Matsumoto, T.: Fatigue crack growth analysis of fiber reinforced concrete with effect of interfacial bond degradation. Cem. Concr. Compos 20, 339-351 (1998)

Loo, K.Y.M., Foster, S.J., Smith, S.T.: FE modeling of CFRP-repaired RC beams subjected to fatigue loading. J. Compos. Constr. 16, 572-580 (2012)

Loo, K.Y.M., Foster, S.J., Smith, S.T.: Fatigue behaviour of CFRP-repaired corroded RC beams (2010)

Lu, X.Z., Teng, J.G., Ye, L.P., Jiang, J.J.: Bond-slip models for FRP sheet/plate-to-concrete interfaces. In: Proceedings of 2nd International Conference of Advanced Polymer Composites for Structural Applications in Construction (ACIC2004), pp. 152-161. Woodhead Publishing Limited, Cambridge (2004)

Lu, X.Z., Teng, J.G., Ye, L.P., Jiang, J.J.: Bond-slip models for FRP sheets/plates bonded to concrete. Eng. Struct. 27, 920-937 (2005)

Maekawa, K., Okamura, H., Pimanmas, A.: Non-linear Mechanics of Reinforced Concrete. CRC Press, Boca Raton (2003)

Matsui, S.: Fatigue strength of RC-slabs of highway bridge by wheel running machine and influence of water on fatigue. Proc. JCI 9, 627-632 (1987)

Mitamura, H., Omote, S., Nishi, H.: Effects of retrofit with two CFRP materials of different properties on fatigue durability improvement of RC slabs, CERI Monthly Report: 2-14 (2011)

Perdikaris, P.C., Beim, S.: RC bridge decks under pulsating and moving load. J. Struct. Eng. 114, 591-607 (1988)

Rots, J.G., Blaauwendraad, J.: Crack models for concrete, discrete or smeared? Fixed, multi-directional or rotating? HERON 34(1), 1989 (1989)

Shakushiro, K., Mitamura, H., Watanabe, T., Kishi, N.: Experimental study on fatigue durability of RC slabs rein-forced with round steel bars. J. Struct. Eng. JSCE 57(A), 1297-1304 (2011). (in Japanese)

Yun, Y., Yu-Fei, W., Tang, W.C.: Performance of FRP bonding systems under fatigue loading. Eng. Struct. 30, 3129-3140 (2008)

Zhao, J., Sritharan, S.: Modeling of strain penetration effects in fiber-based analysis of reinforced concrete structures. ACI Struct. J. 104, 133 (2007) 